International Journal of Biomedicine I June 2019 - Volume 9, Issue Suppl_1: Abstracts From the Second Russian International Conference "Cryo-electron microscopy 2019: achievements and prospects"

POSTER ABSTRACT PRESENTATIONS

SESSION TITLE: APPLICATIONS OF CRYO-EM IN MEDICINE

DOI: 10.21103/IJBM.9.Suppl_1.P37

\title{
Abstract P-37: Ultrastructural Differential-Diagnostic Criteria for Renal Oncocytoma and Chromophobe Renal Cell Carcinoma
}

Elena Smirnova, Irina Bukaeva, Vera Delektorskaya, Svetlana Bezhanova, Alena Puchkova

N. N. Blokhin Russian Cancer Research Center, Ministry of Health of Russia, Moscow, Russia

Background: The differential diagnosis between renal oncocytoma $(\mathrm{RO})$ and chromophobe renal cell carcinoma (chRCC) is clinically significant because of their different biological behavior: the former widely accepted as a benign neoplasm and the latter largely considered as malignant tumor with potential for metastatic spread. The light microscopy differentiation of RO and chRCC may be problematic due to the overlapping morphology among these neoplasms and further investigations are needed to define the correct diagnosis. The aim of this study was to investigate the ultrastructural peculiarities of RO and chRCC and to examine the value of electron microscopy in differentiating between these neoplasms.

Methods: Fifteen histological confirmed cases of RO and 16 cases of chRCC were examined by electron microscopy. Ultrathin sections were used for observation under a JEM-1200EX (II) transmission electron microscopy.

Results: Ultrastructurelly, RO consisted of large clear cells. Nuclei were generally round with smooth margin. Chromatin was finely dispersed, the nucleoli were predominantly centrally located. The cytoplasm was densely packed with numerous mitochondria with lamellar cristae. ChRCC consisted of solid growth pattern of polygonal cells with prominent cell membrane and separated by thin-walled blood vessels. The tumor cell nuclei were polygonal, often with invaginations. Small clumps of condensed chromatin were detected. In the cytoplasm large amounts of glycogen were observed. Mitochondria were polymorphic, showing tubulo-vesicular cristae, part of those were ring-shaped.

Tumor cells of chRCC showed unique feature such as presence of numerous cytoplasmic microvesicles along with mitochondria displaying tubulo-vesicular cristae.

Conclusion: Ultrastructural examination of mitochondria is the helpful approach in the diagnosis of RO and chCRCC: RO showed mitochondria with lamellar cristae while chRCC exhibited mitochondria with tubulo-vesicular cristae.Thus, electron microscopy provides the important information for the differential diagnosis between RO and chRCC, for the estimation of grade malignancy and also for the choice of the appropriate therapy of these tumors.

Key Words: oncocytoma $\bullet$ chromophobe renal cell carcinoma $\bullet$ electron microscopy 
International Journal of Biomedicine. 2019;9 Suppl 1: S33. doi: 10.21103/IJBM.9.Suppl_1.P37 (C)2019 International Medical Research and Development Corporation 